\title{
Positron Annihilation Induced Auger Electron Spectroscopy
}

\author{
Alex Weiss, A.R. Koymen, David Mehl, K.O. Jensenª, Chun Lei and K. H. Lee \\ Physics Department, The University of Texas at Arlington, Arlington Texas 76019
}

\begin{abstract}
Recently, Weiss et al. have demonstrated that it is possible to excite Auger transitions by annihilating core electrons using a low energy (less than $30 \mathrm{eV}$ ) beam of positrons. This mechanism makes possible a new electron spectroscopy, Positron annihilation induced Auger Electron Spectroscopy (PAES). The probability of exciting an Auger transition is proportional to the overlap of the positron wavefunction with atomic core levels. Since the Auger electron energy provides a signature of the atomic species making the transition, PAES makes it possible to determine the overlap of the positron wavefunction with a particular element. PAES may therefore provide a means of detecting positron-atom complexes. Measurements of PAES intensities from clean and adsorbate covered $\mathrm{Cu}$ surfaces are presented which indicate that $\sim 5 \%$ of positrons injected into $\mathrm{Cu}$ at $25 \mathrm{eV}$ produce core annihilations that result in Auger transitions.
\end{abstract}

\section{Introduction}

The Auger process is a nonradiative transition in which an atom with a inner shell hole relaxes by filling this hole with an less tightly bound electron while simultaneously emitting another electron (the Auger electron) which carries off the excess energy. The energy of the Auger electron is given by the equation, $\mathrm{E}_{\mathrm{XYZ}}=\mathrm{E}_{\mathrm{X}}-\mathrm{E}_{\mathrm{Y}}{ }^{*}-\mathrm{E}_{\mathrm{Z}}{ }^{*}$ where $\mathrm{E}_{\mathrm{X}}$ is the binding energy of the electron removed to form the original inner shell hole, and $\mathrm{EY}^{*}, \mathrm{EZ}_{\mathrm{Z}}{ }^{*}$ are the binding energies associated with the two hole final state. Because the energy levels of different elements are in general unique, the elemental identity of an atom may be deduced from the energies of the Auger electrons emitted as a result of core hole excitations. This fact along with the short escape depth of low energy electrons has been exploited in the widely used surface analysis tool, Auger Electron Spectroscopy (AES).

Conventional Electron induced Auger Electron Spectroscopy (EAES) makes use of high energy electrons to collisionally ionized the atom. However in many instances the utility of EAES is limited by problems associated with the large secondary electron background and the lack of surface specificity inherent in the EAES excitation process. Recently, Weiss et al. ${ }^{1}$ have demonstrated that Auger electrons can be excited with high efficiency by using low energy positrons to produce the core hole excitations necessary for Auger electron emission by matter - antimatter annihilation. This process makes possible a new surface spectroscopy, Positron annihilation induced Auger Electron Spectroscopy (PAES) which has significant advantages over conventional EAES in some systems. In the remainder of the paper we will describe experiments which demonstrate the potential advantages of the PAES technique. We the describe theoretical calculations from which we estimate the efficiency with which positrons induce Auger transitions. This estimate is then compared to experimental values. The paper concludes with a discussion of the possible use of PAES to detect positron -atom or positron - molecule bound states

Elimination of Secondary Electron Background The PAES technique can be used to eliminate the large secondary electron background that limits the sensitivity and accuracy of conventional methods of Auger Electron Spectroscopy (AES). ${ }^{1-3}$ In PAES, the core hole excitations necessary for AES are generated by matter-antimatter annihilation and not by collisional processes. It is therefore possible to use an incident beam energy well below the Auger electron energy thus precluding the creation of secondary electrons in the energy range of the Auger signal. In contrast, in conventional electron 
stimulated Auger electron spectroscopy, (EAES), the incident beam energy must be in excess of the Auger electron energy which makes it impossible to avoid creating a large secondary electron background. The large improvement in signal to background that can be obtained using PAES is demonstrated in Figure 1. which compares Auger spectra obtained using positron excitation to that using conventional EAES. Both spectra were obtained using the UTA positron Auger system. Signal to background levels of greater than 40:1 were obtained (more than a factor of 80 improvement over conventional methods of AES). The improved signal-to -background allows PAES data to be taken with beam currents several orders of magnitude less than in conventional electron excited Auger (EAES). The low currents and low beam energies used in PAES allow the energy dose require to obtain data to be reduced four to six


Fig. 1. Comparison of Conventional EAES and PAES spectra. Note the large increase in signal to background for the $\mathrm{Cu} \mathrm{M}_{2,3} \mathrm{VV}$ peak obtained using PAES. orders of magnitude as compared to EAES. This will permit the use of PAES in fragile systems where conventional methods of AES are severely limited.

Surface Selectivity: Positron annihilation induced Auger spectroscopy displays enhanced surface selectivity. 3,4 This selectivity is due to the restriction of the excitation volume to the top atomic layer due to localization of the positron. This is in contrast to conventional Auger in which the excitation volume extends hundreds of atomic layer below the surface. EAES acquires its surface sensitivity solely from the 4-20A escape depth of the Auger electron. The intensity of the $\mathrm{Cu} \mathrm{M}_{2,3} \mathrm{VV}$ PAES signal decrease by a factor of 4 with the addition of a $1 / 2$ monolayer of $S$ on the surface (see figure 2). This is contrasted with only a $25 \%$ decrease in the EAES signal caused by the overlayer. These results were accounted for by theoretical calculations which show that the positron wavefunction is pushed away from the $\mathrm{Cu}$ surface causing the decrease in PAES intensity. These same calculations demonstrate that as much as $97 \%$ of the Auger signal will originate in the top atomic layer using PAES as compared to about $50 \%$ using conventional AES techniques.

Theoretical Calculations: Theoretical calculations were carried out ${ }^{5}$ to determine the expected magnitude of PAES intensities. In addition, detailed surface calculations were carried out in support of our experimental measurements of the surface selectivity for PAES results to determine the spatial extent of the positron wavefunction and the degree of surface selectivity that could be attained with PAES. 3,4 Calculations using a corrugated mirror model for the positron surface potential were performed on clean metal and overlayer on metal surfaces producing good agreement with experimental results. ${ }^{3}$

Nearly all $\mathrm{Cu} 3 \mathrm{p}$ holes decay via emission of an $\mathrm{M}_{2,3} \mathrm{VV}$ Auger electron (energy $\approx 60 \mathrm{eV}$ ) since the radiative transition probability is extremely small. 5 For clean $\mathrm{Cu}(100)$ and $\mathrm{Cu}(110)$ the annihilation probabilities for the $3 \mathrm{~s}$ and $3 \mathrm{p}$ electrons are calculated to be $\mathrm{p}_{3 \mathrm{~s}}=0.83 \%$ and $\mathrm{p}_{3 \mathrm{p}}=3.0 \%$, respectively. Putting in the relevant Auger transiton rates we estimate that, $\sigma_{\mathrm{M} 23 \mathrm{VV}}$, the probability of a positron trapped in a surface state causing the a $M_{2,3} \mathrm{VV}$ Auger transition is $-3.6 \%$. The annihilation probabilities for the deeper lying $2 \mathrm{~s}$ and $2 p$ levels are two orders of magnitude lower. The calculations also indicate that core annihilations take 
place primarily in the top surface layer, with about $5 \%$ and $20 \%$ of the total rate arising from second or deeper layers for $\mathrm{Cu}(100)$ and $\mathrm{Cu}(110)$, respectively.

For the purpose of making a comparison with theoretical calculations, we estimate $\sigma \mathrm{M} 23 \mathrm{VV}$ as follows 6 : an integral over the energy spectrum of the PAES $\mathrm{Cu} \mathrm{M} 2,3 \mathrm{VV}$ Auger peak was compared

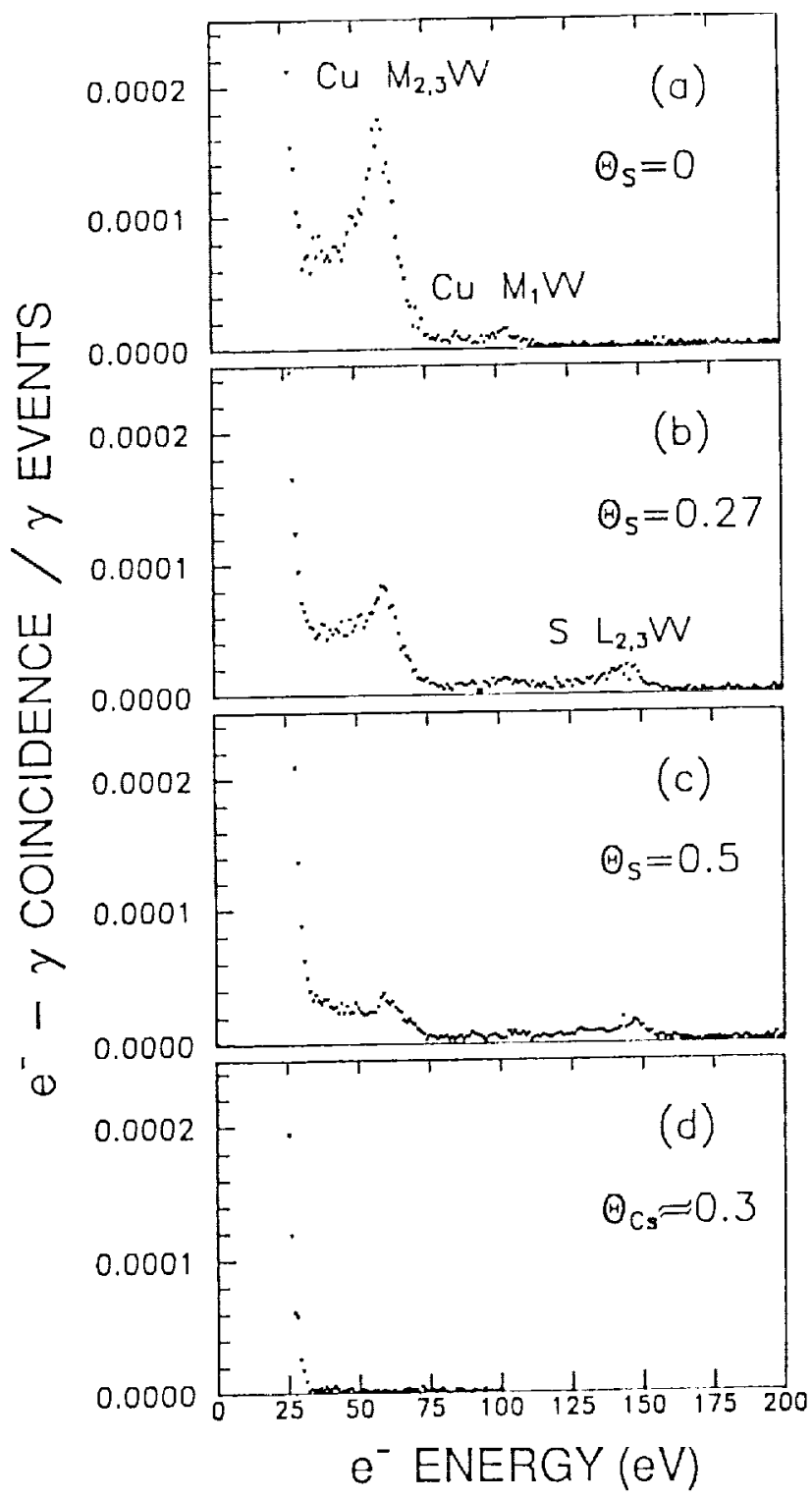

Figure 2. PAES spectra obtained from clean and adsorbate covered $\mathrm{Cu}$. The large decrease in the $\mathrm{Cu}$ signal with overlayer coverage demonstrates the top layer selectivity of PAES. to an integral over the positron induced secondary electron peak. The secondary electron peak was obtained with the sample at $-60 \mathrm{~V}$, so the electrons pass through the spectrometer at approximately the same energy as the Auger electrons. The positrons were incident with a kinetic energy of $80 \mathrm{eV}$. Measurements of positron induced secondary electron emission from $\mathrm{Ni}$ at an angle of $50^{\circ}$ to normal incidence allowed an estimate of the ratio of secondary electrons per incident positron, $\delta$, in this experiment. The measured ratio of the Auger yield to the secondary yield was then substituted into a formula 6 which takes into account detector solid angle and efficiencies to give: $\sigma_{\mathrm{m}_{2,3}}=5.6 \%$. Part of the discrepancy between this value and the theoretically calculated value of $3.6 \%$ may be due to neglect of the many-body enhancement factor.

It is interesting to speculate on the possibility using PAES as a means of signaling the existence of a positron-atom or positron-molecule bound state. Since the overlap of the positron wavefunction with the core levels of an atom should be enhanced if the positron were bound to that atom, the existence of a bound state would be signaled by an increase in the PAES intensity. It may be possible to test this hypothesis by using a very low energy beam of positrons incident on atoms or molecules physisorbed on a metallic substrate.

This research was supported in part by the Texas Advanced Research Program and the Robert A. Welch Foundation.

a. Permanent address: Physics Department, The University East Anglia, Norwhich, UK

\section{References}

1. Weiss, Mayer, Mehl,Jibaly, Lei, and Lynn, Phys. Rev. Lett. 61, 2245(1988).

2. Weiss, Mehl, Lei, Jibaly, Mayer and Lynn, accepted for publication in Positron Annihilation, World Scientific Press, Singapore (1989).

3. Mehl, Koymen, Jensen, Gotwald and Weiss, submitted to Phys. Rev. Lett.

4. Lei, Mehl, Koymen, Jibaly, Gotwald and Weiss, UTA preprint.

5. Jensen and Weiss, UTA preprint.

6. David Mehl, Thesis (unpublished) 